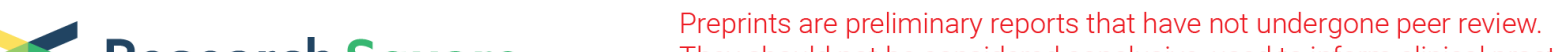 Research Square
or referenced by the media as validated information.
}

\section{Prognostic Value of Systemic Inflammatory Markers and the Nutrition Status in Thymic Epithelial Tumors with Complete Resection}

\section{Tadashi Sakane}

Nagoya City University Graduate School of Medical Sciences and Medical School: Nagoya Shiritsu Daigaku Daigakuin Igaku Kenkyuka Igakubu

Katsuhiro Okuda ( $\square$ kokuda@med.nagoya-cu.ac.jp )

Nagoya City University Graduate School of Medical Sciences https://orcid.org/0000-0003-0099-2348

\section{Takuya Matsui}

Nagoya City University Graduate School of Medical Sciences and Medical School: Nagoya Shiritsu Daigaku Daigakuin Igaku Kenkyuka Igakubu

\section{Risa Oda}

Nagoya City University Graduate School of Medical Sciences and Medical School: Nagoya Shiritsu Daigaku Daigakuin Igaku Kenkyuka Igakubu

\section{Tsutomu Tatematsu}

Nagoya City University Graduate School of Medical Sciences and Medical School: Nagoya Shiritsu Daigaku Daigakuin Igaku Kenkyuka Igakubu

\section{Keisuke Yokota}

Nagoya City University Graduate School of Medical Sciences and Medical School: Nagoya Shiritsu Daigaku Daigakuin Igaku Kenkyuka Igakubu

\section{Ryoichi Nakanishi}

Nagoya City University Graduate School of Medical Sciences and Medical School: Nagoya Shiritsu Daigaku Daigakuin Igaku Kenkyuka Igakubu

\section{Research article}

Keywords: thymic epithelial tumor, neutrophil-to-lymphocyte ratio, monocyte-to-lymphocyte ratio, prognostic nutritional index

Posted Date: June 16th, 2021

DOl: https://doi.org/10.21203/rs.3.rs-618470/v1

License: (a) This work is licensed under a Creative Commons Attribution 4.0 International License. Read Full License 


\section{Abstract}

\section{Background}

Recent studies have shown that several systemic inflammatory markers and the nutrition status, including the neutrophil-to-lymphocyte ratio (NLR), monocyte-to-lymphocyte ratio (MLR), platelet-tolymphocyte ratio (PLR), and prognostic nutritional index (PNI), are useful prognostic factors in several malignant tumors. The present study explored the prognostic value of the NLR, MLR, PLR, and PNI in thymic epithelial tumor (TET) patients who underwent complete resection.

\section{Methods}

A total of 158 TET patients who underwent complete resection were involved in the analysis. Their NLR, MLR, PLR, and PNI values were obtained from a blood examination within one month before the initiation of treatment. A receiver operating characteristic curve analysis was conducted to determine the optimal cut-off values.

\section{Results}

The enrolled patients were stratified by cut-offs of 4.35 for the NLR, 0.22 for the MLR, 130.18 for the PLR, and 44.02 for the PNI. A univariate analysis revealed that high-grade malignant TET, including type B2 and B3 thymoma, thymic carcinoma, and thymic neuroendocrine tumor; an advanced Masaoka stage; a high NLR; a high MLR; and a low PNI were significant predictors of a poor disease-free survival (DFS). A multivariate analysis confirmed that an advanced Masaoka stage $(H R=5.5557, P=0.0007)$ and a high $\operatorname{MLR}(H R=3.3371, P=0.0264)$ were independent predictors of a poor DFS.

\section{Conclusions}

Our study demonstrated that the pretreatment MLR was an independent predictor of the DFS in patients with TETs who underwent complete resection.

\section{Background}

Thymic epithelial tumors (TETs), including thymomas, thymic carcinomas, and thymic neuroendocrine tumors, are rare tumors derived from thymic epithelium. TETs are heterogenous genetically and histopathologically $[1,2]$. Currently, the Masaoka stage or pathological TNM stage is considered the best predictor of the long-term survival of TET patients; however, such values are confirmed only after surgery. In order to provide appropriate treatment, specific biomarkers that can predict the prognosis and therapeutic response before treatments are desired.

The survival is also determined by host-related factors, including systemic inflammatory markers and the nutrition status. Recent studies have clarified that tumor-related immune responses are significantly related to tumor progression [3]. Cytokines produced by tumor cells or tumor microenvironment can 
stimulate the host inflammation, which leads to an increase in circulating peripheral blood cells, including neutrophils, lymphocytes, monocytes, and platelets [4]. Since Virchow first noted leukocytes in neoplastic tissues and discussed the relationship between inflammation and cancers in 1881, the peripheral blood neutrophil-to-lymphocyte ratio (NLR), monocyte-to-lymphocyte ratio (MLR), and platelet-to-lymphocyte ratio (PLR) have been widely used to predict the prognosis of cancers, including gastric cancer, colorectal cancer, lung cancer, esophageal cancer, and breast cancer [5-9]. Similarly, the prognostic nutritional index (PNI) proposed by Onodera et al. is a prognostic index that reflects both the nutritional and immunological statuses, and a low PNI has been reported to predict a poor survival in various types of cancer [10-12].

Thus far, only a few studies have reported a relationship between systemic inflammatory markers or the nutrition status and the prognosis of TET patients. The present study therefore explored the prognostic value of the NLR, MLR, PLR, and PNI in 158 TET patients who underwent complete surgical resection.

\section{Methods}

\section{Study population}

This retrospective study was approved by the Institutional Review Board at Nagoya City University Hospital (Nagoya, Japan) (approval number 70-00-0038). We reviewed the medical records of patients with TET, who underwent complete surgical resection from April 2004 to December 2019 at Nagoya City University Hospital. Patients were excluded according to the following criteria: I) had recent steroid therapy, II) had active infection or other bone marrow disorders, III) had blood transfusion, IV) had a treatment history of another type of cancer within one year, $\mathrm{V}$ ) had recurrence or incomplete resection, and $\mathrm{VI}$ ) had incomplete clinicopathological or examination data or follow-up information.

\section{Definition of inflammatory markers and the survival}

Data were compiled from individual patient medical case notes, electronic patient record, and pathology reports. Peripheral venous blood samples were collected within one month before the initiation of treatment. The NLR was derived by dividing the absolute neutrophil count by the absolute lymphocyte count in peripheral blood. The MLR and PLR were calculated in a similar manner. The PNI was calculated as $10 \times$ serum albumin value $(\mathrm{g} / \mathrm{dL})+0.005 \times$ peripheral lymphocyte count $\left(\right.$ per $\left.\mathrm{mm}^{3}\right)$. To determine the optimal cut-off value for each inflammatory marker, a receiver operating characteristic (ROC) curve was generated to predict tumor recurrence. The maximum Youden index indicated the optimum cut-off. The disease-free survival (DFS) time was defined as the interval between the operation and the first incidence of detectable recurrence. The overall survival (OS) time was defined as the interval between the operation and death or the last follow-up.

Statistical analyses 
The optimal cut-off values of the NLR, MLR, PLR, and PNI for the prediction of recurrence were determined with an ROC curve analysis according to the maximum Youden index. Survival curves were generated using the Kaplan-Meier method, and the log-rank test was used to assess the statistical significance of differences between the two groups. A Cox proportional hazards model was used to estimate the hazard ratios and $95 \%$ confidence intervals. The prognostic variables identified by a univariate analysis were further analyzed in a multivariate Cox proportional hazards model. A two-sided P-value of $<0.05$ was considered to indicate a statistically significant difference. All of the statistical analyses in this study were performed using the JMP software program (version 12.0.1; SAS Institute, Cary, NC, USA).

\section{Results}

\section{Patients' characteristics}

A total of 158 TET patients who underwent complete resection were included in the study. The clinicopathological variables of the included patients are presented in Table 1. The study cohort included 84 men and 74 women, with a median age of 61 (range: 24-87) years old. Autoimmune disease was found in 27 patients, all of whom had thymoma. Eight patients who received preoperative steroid pulse therapy had thymoma. Two patients with thymic carcinoma and one with type B2 thymoma received preoperative chemotherapy, and one patient with thymic carcinoma received preoperative chemoradiotherapy. The histological subtypes of the 20 cases who received adjuvant therapy were as follows: type $A$ thymoma, $n=1$; type B1 thymoma, $n=1$; type B2 thymoma, $n=4$; type B3 thymoma, $n=$ 2 ; thymic neuroendocrine tumor, $n=1$; thymic carcinoma, $n=11$. The median follow-up time was 61 (range, 0-174) months. The clinical courses of all patients were as follows: alive and well, $n=134$; alive with disease, $n=14$; died of other disease, $n=6$; and die of disease, $n=4$.

\section{ROC curves for the predictions of recurrence}

In this study, we performed ROC analyses to select appropriate cut-off values for predicting recurrence for the inflammatory markers (Figure 1). The cut-off value was 4.35 for the preoperative NLR, with an area under the curve (AUC) of 0.604 . The preoperative NLR had a sensitivity of $27.78 \%$ and a specificity of $94.29 \%$ for predicting recurrence. The best cut-off values for the preoperative PLR and MLR were 0.22 and 130.18 , respectively, with AUCs of 0.618 (sensitivity $=61.11 \%$, specificity $=75.71 \%$ ) and 0.495 (sensitivity $=66.67$, specificity $=47.86 \%$ ). The cut-off value was 44.02 for the preoperative PNI, with an AUC of 0.591 (sensitivity $=72.22 \%$, specificity $=53.57 \%$ ).

\section{Inflammatory markers and the survival}

Kaplan-Meier curves demonstrated that a high NLR $(\geq 4.35)(p=0.0009)$, a high MLR $(\geq 0.22)(P=$ $0.0001)$, and a low PNI $(<44.02)(P=0.0426)$ were associated with significantly lower DFS rates than a low NLR (<4.35), a low MLR (<0.22), and a high PNI ( $\geq 44.02)$, respectively (Figure 2). The PLR did not predict the DFS $(P=0.4100)$. The univariate analysis revealed that high-grade malignant TET, including 
type B2 and B3 thymoma, thymic carcinoma, and thymic neuroendocrine tumor (hazard ratio $[\mathrm{HR}]=$ 8.0671, $P=0.0004)$; an advanced Masaoka stage $(H R=7.9015, P<0.0001)$; a high NLR $(H R=4.8511, P$ $=0.0091)$; a high MLR $(H R=5.1991, P=0.0006)$; and a low PNI $(H R=2.7766, P=0.0398)$ were significant predictors of a poor DFS (Table 2). A subsequent multivariate analysis confirmed that an advanced Masaoka stage $(H R=5.5557, P=0.0007)$ and a high $M L R(H R=3.3371, P=0.0264)$ were independent predictors of a poor DFS (Table 2). No inflammatory markers predicted the OS after surgery (data not shown).

\section{Discussion}

In the present study, we explored the prognostic value of the pretreatment NLR, MLR, PLR and PNI in 158 patients with completely resected TETs. We found that, according to the univariate analysis, an advanced Masaoka stage, increased NLR, increased MLR, and decreased PNI were prognostic factor for a poor DFS. The subsequent multivariate analysis revealed that an advanced Masaoka stage and increased MLR were independent prognostic factors for recurrence.

The relationship between the NLR or MLR and prognosis appears to be complicated, and the precise mechanisms involved are not fully understood. An increased NLR or MLR indicates elevated neutrophils, elevated monocytes, or decreased lymphocytes. The systemic inflammatory response from cancer cells promotes the infiltration of neutrophils, which benefits cancer progression via the secretion of interleukin (IL)-2, IL-6, IL-10, tumor necrosis factor a (TNF-a), and vascular endothelial growth factor (VEGF) [13]. Neutrophilia as an inflammatory response inhibits the immune system by suppressing the cytokine activity of immune cells, such as lymphocytes, activated T-cells, and natural killer cells $[14,15]$. Monocytes also play an important role in malignancies, as they interact with adaptive immunity by directing the recruitment and function of lymphocytes within the tumor microenvironment [16]. Circulating monocytes take part in paracrine signaling and induce increases in the levels of many inflammatory cytokines and chemokines, including IL-1, IL-6, TNF- $a$, and chemokine ligand 3 [17]. In addition, tumor-associated macrophages, which originate from circulating monocytes, enhance the pro-tumoral functions, including tumor cell migration, invasion, metastasis, and angiogenesis, and suppress the immune reaction against tumor cells [18-20]. Lymphocytes also play an important role in malignancies. Lymphocytes serve a fundamental role in antitumor immunity [21]. The increasing infiltration of tumors with lymphocytes is reportedly associated with an improved response to cytotoxic treatment and the prognosis in patients with cancer [21,22]. These factors are said to be involved in the tumor microenvironment and associated with carcinogenesis, proliferation, and infiltration of cancer cells.

In recent years, many studies have reported that a high NLR or MLR is associated with a poor survival in patients with various malignant tumors, including gastric cancer, colorectal cancer, lung cancer, esophageal cancer, and breast cancer [5-9]. To our knowledge, there have been only five reports examining the association of the NLR with the prognosis of TETs, and the present one is the secondlargest study [23-27]. The cut-off values used in those reports ranged from 1.96 to 4.10, and the value used in the present study was 4.3 , which was similar to the values in the previous five reports. In all five 
reports, a high NLR was associated or tended to be associated with a poor prognosis. Our finding that a high NLR was associated with a shorter DFS supports these previous findings. Among those five reports, the one by Wang et al. described the relationship between the pretreatment MLR and prognosis. Those authors found that a high MLR was associated with tumor aggressiveness, and a co-high maximum standardized uptake value $\left(S U V_{\text {max }}\right) / M L R$ was an independent risk factor for recurrence [27]. In the present study, an increased pretreatment MLR was found to be an independent predictor of recurrence. There were 11 cases of recurrence with increased pretreatment MLR values, including 5 with local recurrence and 6 with dissemination. Among these 11 cases, 3 were Masaoka stage II, 4 were stage III, and the remaining 4 were stage IV. Four cases, including two stage II cases, one stage III case, and one stage IV case, had not received neoadjuvant or adjuvant therapy. We believe that patients with increased pretreatment MLR values should receive neoadjuvant or adjuvant therapy even if they are at an early Masaoka stage.

The PNI is an index reflecting the systemic immune-nutritional status of patients. Large amounts of evidence indicate that higher PNI values are associated with a better survival in malignancies [28-30]. The $\mathrm{PNI}$ is initially designed to assess the perioperative nutritional status and predict perioperative complications $[10,31]$. While the serum albumin level is used to assess the nutritional status and immune function, a reduced albumin level is associated with tumor progression, metastasis, and increased risk of death after surgery [32]. To our knowledge, this is the first report to describe the predictive value of the PNI in TET patients. The optimal cut-off value of the PNI to predict the prognosis of patients with TETs has not been investigated before, so we conducted a ROC curve analysis to explore the optimal cut-off value of the PNI among TET patients. Our results were in line with those of previously published studies describing a significant relationship between the PNI and prognosis of cancers.

Several limitations associated with the present study warrant mention. First, this was a retrospective study conducted in a single institution, although it was a relatively large-scale $(n=158)$ study to evaluate the prognostic value of pretreatment NLR, MLR, PLR, and PNI. It may not be possible to completely avoid selection and information bias. Further studies including more patients from multiple centers are needed to validate the results. Second, we used the cut-off values determined based on ROC curves. At present, there are no established cut-off values. Future studies should endeavor to establish optimal cut-off values specific to TETs. Third, we used only the baseline values of the NLR, MLR, PLR, and PNI, rather than accounting for dynamic changes in each systemic inflammatory marker and nutrition status. In addition, various factors, including other disease conditions and medications, were not considered. Fourth, we found no significant relationship between the systemic inflammatory markers or nutrition status, including the NLR, MLR, PLR, and PNI, and the OS. In the present study, the median follow-up time was 61 months. Patients with thymomas, which account for the majority of TETs, are expected to survive for a relatively long period of time. A much longer follow-up period is needed to examine the association of the systemic inflammatory markers and the nutrition status with the OS.

\section{Conclusions}


In conclusion, our study showed that the MLR was an independent predictor of a poor DFS in patients with TETs who underwent complete resection. If our results are validated in future studies, the MLR may be recognized as a valuable biomarker for predicting the prognosis in TET patients.

\section{Abbreviations}

TET: Thymic epithelial tumors; NLR: Neutrophil-to-lymphocyte ratio; MLR: Monocyte-to-lymphocyte ratio; PLR: Platelet-to-lymphocyte ratio; PNI: Prognostic nutritional index; ROC: Receiver operating characteristic; DFS: Disease-free survival; OS: Overall survival; AUC: Area under the curve; HR: Hazard ratio; IL: Interleukin; TNF-a: Tumor necrosis factor a; VEGF: Vascular endothelial growth factor; SUV: Standardized uptake value

\section{Declarations}

\section{Ethics approval and consent to participate}

This study was approved by the Institutional Review Board at Nagoya City University Hospital (Nagoya, Japan) (approval number 70-00-0038), and it conforms to the provisions of the Declaration of Helsinki.

\section{Consent for publication}

Not applicable.

\section{Availability of data and material}

The data that support the findings of this study are not publicly available to respect individual privacy of the patients included, but are available from the corresponding author upon reasonable request.

\section{Competing interests}

All authors declare that they have no competing interest.

\section{Funding}

Not applicable

\section{Authors' contributions}

$\mathrm{RN}, \mathrm{KO}$, and TS designed the study and prepared the manuscript. TS, TM, and OR collected, analyzed, and interpreted the data. TT and KY overviewed the quality of data and reviewed the manuscript. All authors have read and approved the manuscript.

\section{Acknowledgements}


Not applicable

\section{References}

[1] Radovich M, Pickering CR, Felau I, Ha G, Zhang H, Jo H, et al. The Integrated Genomic Landscape of Thymic Epithelial Tumors. Cancer Cell. 2018; 33: 244-58.

[2] Conforti F, Pala L, Giaccone G, Pas TD. Thymic epithelial tumors: From biology to treatment. Cancer Treat Rev. 2020; 86: 102014.

[3] Grivennikov SI, Greten FR, Karin M. Immunity, inflammation, and cancer. Cell. 2010; 140: 883-99.

[4] Valero C, Pardo L, López M, García J, Camacho M, Quer M, et al. Pretreatment count of peripheral neutrophils, monocytes, and lymphocytes as independent prognostic factor in patients with head and neck cancer. Head Neck. 2017; 39: 219-26.

[5] Kim EY, Song KY. The preoperative and the postoperative neutrophil-to-lymphocyte ratios both predict prognosis in gastric cancer patients. World J Surg Oncol. 2020; 18: 293.

[6] Haram A, Boland MR, Kelly ME, Bolger JC, Waldron RM, Kerin MJ. The prognostic value of neutrophilto-lymphocyte ratio in colorectal cancer: A systematic review. J Surg Oncol. 2017; 115: 470-9.

[7] Diem S, Schmid S, Krapf M, Flatz L, Born D, Jochum W, et al. Neutrophil-to-Lymphocyte ratio (NLR) and Platelet-to-Lymphocyte ratio (PLR) as prognostic markers in patients with non-small cell lung cancer (NSCLC) treated with nivolumab. Lung Cancer. 2017; 111: 176-81.

[8] Barbetta A, Nobel TB, Sihag S, Hsu M, Tan KS, Bains MS, et al. Neutrophil to Lymphocyte Ratio as Predictor of Treatment Response in Esophageal Squamous Cell Cancer. Ann Thorac Surg. 2018; 106: 86471.

[9] Ethier JL, Desautels D, Templeton A, Shah PS, Amir E. Prognostic role of neutrophil-to-lymphocyte ratio in breast cancer: a systematic review and meta-analysis. Breast Cancer Res. 2017; 19: 2.

[10] Onodera T, Goseki N, Kosaki G. Prognostic nutritional index in gastrointestinal surgery of malnourished cancer patients. Nihon Geka Gakkai Zasshi. 1984; 85: 1001-5.

[11] Ninomiya S, Kawahara T, Miyoshi Y, Yao M, Uemura H. A retrospective study on the possible systematic inflammatory response markers to predict the prognosis of patients with bladder cancer undergoing radial cystectomy. Mol Clin Oncol. 2020; 13: 47.

[12] Sun K, Chen S, Xu J, Li G, He Y. The prognostic significance of the prognostic nutritional index in cancer: a systematic review and meta-analysis. J Cancer Res Clin Oncol. 2014; 140: 1537-49.

[13] Balkwill F, Mantovani A. Inflammation and cancer: back to Virchow? Lancet. 2001; 357:539-45. 
[14] Petrie HT, Klassen LW, Kay HD. Inhibition of human cytotoxic T lymphocyte activity in vitro by autologous peripheral blood granulocytes. J Immunol. 1985; 134: 230-4.

[15] el-Hag A, Clark RA. Immunosuppression by activated human neutrophils. Dependence on the myeloperoxidase system. J Immunol. 1987; 139: 2406-13.

[16] Jakubowska K, Koda M, Grudzińska M, Kańczuga-Koda L, Famulski W. Monocyte-to-lymphocyte ratio as a prognostic factor in peripheral whole blood samples of colorectal cancer patients. World $\mathrm{J}$ Gastroenterol. 2020; 26: 4639-55.

[17] Chittezhath M, Dhillon MK, Lim JY, Laoui D, Shalova IN, Teo YL, et al. Molecular profiling reveals a tumor-promoting phenotype of monocytes and macrophages in human cancer progression. Immunity. 2014; 41: 815-29.

[18] Coussens LM, Werb Z. Inflammation and cancer. Nature. 2002; 420: 860-7.

[19] Condeelis J, Pollard JW. Macrophages: obligate partners for tumor cell migration, invasion, and metastasis. Cell. 2006; 124: 263-6.

[20] Pollard JW. Tumour-educated macrophages promote tumour progression and metastasis. Nat Rev Cancer. 2004; 4: 71-8.

[21] Gooden MJ, de Bock GH, Leffers N, Daemen T, Nijman HW. The prognostic influence of tumourinfiltrating lymphocytes in cancer: a systematic review with meta-analysis. $\mathrm{Br} J$ Cancer. 2011; 105: 93103.

[22] Denkert C, Loibl S, Noske A, Roller M, Müller BM, Komor M, et al. Tumor-associated lymphocytes as an independent predictor of response to neoadjuvant chemotherapy in breast cancer. J Clin Oncol. 2010; 28: 105-13.

[23] Yuan ZY, Gao SG, Mu JW, Xue Q, Mao YS, Wang DL, et al. Prognostic value of preoperative neutrophillymphocyte ratio is superior to platelet-lymphocyte ratio for survival in patients who underwent complete resection of thymic carcinoma. J Thorac Dis. 2016; 8: 1487-96.

[24] Yanagiya M, Nitadori J, Nagayama K, Anraku M, Sato M, Nakajima J. Prognostic significance of the preoperative neutrophil-to-lymphocyte ratio for complete resection of thymoma. Surg Today. 2018; 48 : 422-30.

[25] Janik S, Raunegger T, Hacker P, Ghanim B, Einwallner E, Müllauer L, et al. Prognostic and diagnostic impact of fibrinogen, neutrophil-to-lymphocyte ratio, and platelet-to-lymphocyte ratio on thymic epithelial tumors outcome. Oncotarget. 2018; 9: 21861-75.

[26] Muriana P, Carretta A, Ciriaco P, Bandiera A, Negri G. Assessment of the prognostic role of neutrophilto-lymphocyte ratio following complete resection of thymoma. J Cardiothorac Surg. 2018; 13: 119. 
[27] Wang L, Ruan M, Yan H, Lei B, Sun X, Chang C, et al. Pretreatment serum neutrophil-to-lymphocyte and monocyte-to-lymphocyte ratios: Two tumor-related systemic inflammatory markers in patients with thymic epithelial tumors. Cytokine. 2020; 133: 155149.

[28] Wang Z, Wang Y, Zhang X, Zhang T. Pretreatment prognostic nutritional index as a prognostic factor in lung cancer: Review and meta-analysis. Clin Chim Acta. 2018; 486: 303-10.

[29] Li S, Tian G, Chen Z, Zhuang Y, Li G. Prognostic Role of the Prognostic Nutritional Index in Pancreatic Cancer: A Meta-analysis. Nutr Cancer. 2019; 71:207-13.

[30] Mirili C, Yılmaz A, Demirkan S, Bilici M, Basol Tekin S. Clinical significance of prognostic nutritional index (PNI) in malignant melanoma. Int J Clin Oncol. 2019; 24: 1301-10.

[31] Kanda M, Fujii T, Kodera Y, Nagai S, Takeda S, Nakao A. Nutritional predictors of postoperative outcome in pancreatic cancer. Br J Surg. 2011; 98: 268-74.

[32] Kanda M, Mizuno A, Tanaka C, Kobayashi D, Fujiwara M, Iwata N, et al. Nutritional predictors for postoperative short-term and long-term outcomes of patients with gastric cancer. Medicine (Baltimore). 2016; 95: e3781.

\section{Tables}


Table 1. Clinical and pathological findings in the cases of thymic epithelial tumor.

\begin{tabular}{|c|c|c|c|}
\hline Factor & & Value & $\%$ \\
\hline Age & median & 61 & \\
\hline \multirow[t]{2}{*}{ Sex } & Male & 84 & 53.2 \\
\hline & Female & 74 & 46.8 \\
\hline Tumor size $(\mathrm{mm})$ & median & 47 & \\
\hline \multirow[t]{7}{*}{ Histological type } & Type A & 12 & 7.6 \\
\hline & Type AB & 35 & 22.2 \\
\hline & Type B1 & 35 & 22.2 \\
\hline & Type B2 & 43 & 27.2 \\
\hline & Type B3 & 13 & 8.2 \\
\hline & Thymic carcinoma & 17 & 10.8 \\
\hline & Thymic neuroendocrine tumor & 3 & 1.9 \\
\hline \multirow[t]{5}{*}{ Autoimmune disease } & Myasthenia gravis & 22 & 13.9 \\
\hline & Pure red cell aplasia & 3 & 1.9 \\
\hline & Good syndrome & 2 & 1.3 \\
\hline & Rheumatoid arthritis & 1 & 0.6 \\
\hline & none & 130 & 82.3 \\
\hline \multirow[t]{4}{*}{ Masaoka classification } & I & 48 & 30.4 \\
\hline & II & 80 & 50.6 \\
\hline & III & 22 & 13.9 \\
\hline & IVa and IVb & 8 & 5.1 \\
\hline
\end{tabular}


Table 2. A univariate and multivariate logistic regression analysis for clinical and pathological features and systemic inflammatory markers.

\begin{tabular}{|c|c|c|c|c|c|c|}
\hline \multirow[t]{2}{*}{ Factor } & \multicolumn{3}{|c|}{ Univariate analysis } & \multicolumn{3}{|c|}{ Multivariate analysis } \\
\hline & $\mathrm{HR}$ & $95 \% \mathrm{Cl}$ & $P$ value & $\mathrm{HR}$ & $95 \% \mathrm{Cl}$ & $P$ value \\
\hline $\begin{array}{l}\text { Age }(\geq 60 \text { years vs. }<60 \\
\text { years })\end{array}$ & 1.4155 & $\begin{array}{l}0.5556- \\
3.7280\end{array}$ & 0.4647 & & & \\
\hline Sex (Male vs. Female) & 0.5525 & $\begin{array}{l}0.2032- \\
1.4038\end{array}$ & 0.2133 & & & \\
\hline $\begin{array}{l}\text { Tumor size ( } \geq 5 \text { cm vs. }<5 \\
\mathrm{~cm})\end{array}$ & 1.6798 & $\begin{array}{l}0.6596- \\
4.5756\end{array}$ & 0.2783 & & & \\
\hline $\begin{array}{l}\text { WHO classification (Type } \\
\text { B2, B3, Carcinoma, } \\
\text { Neuroendocrine tumor vs } \\
\text { Type A, AB, B1) }\end{array}$ & 8.0671 & $\begin{array}{l}2.2933- \\
51.0351\end{array}$ & 0.0004 & 3.8573 & $\begin{array}{l}0.9906- \\
25.5180\end{array}$ & 0.0518 \\
\hline $\begin{array}{l}\text { Masaoka stage (III or IV vs. I } \\
\text { or II) }\end{array}$ & 7.9015 & $\begin{array}{l}3.0950- \\
21.5740\end{array}$ & $<0.0001$ & 5.5557 & $\begin{array}{l}2.0733- \\
15.9668\end{array}$ & 0.0007 \\
\hline NLR ( $\geq 4.35$ vs. $<4.35$ ) & 4.8511 & $\begin{array}{l}1.5545- \\
12.8849\end{array}$ & 0.0091 & 1.7290 & $\begin{array}{l}0.5150- \\
5.1011\end{array}$ & 0.3544 \\
\hline $\operatorname{MLR}(\geq 0.22$ vs. $<0.22)$ & 5.1991 & $\begin{array}{l}2.0454- \\
14.1424\end{array}$ & 0.0006 & 3.3371 & $\begin{array}{l}1.1553- \\
9.8820\end{array}$ & 0.0264 \\
\hline PLR ( $\geq 130.18$ vs. $<130.18)$ & 0.6643 & $\begin{array}{l}0.2311- \\
1.7120\end{array}$ & 0.4045 & & & \\
\hline PNI (<44.02 vs. $\geq 44.02)$ & 2.7766 & $\begin{array}{l}1.0469- \\
8.6557\end{array}$ & 0.0398 & 1.4038 & $\begin{array}{l}0.4926- \\
4.6092\end{array}$ & 0.5366 \\
\hline
\end{tabular}

$\mathrm{HR}$, hazard ratio; $\mathrm{Cl}$, confidence Interval; NLR, neutrophil-lymphocyte ratio; MLR, monocyte-lymphocyte ratio; PLR, platelet-lymphocyte ratio; PNI, prognostic nutritional index.

\section{Figures}


(a)

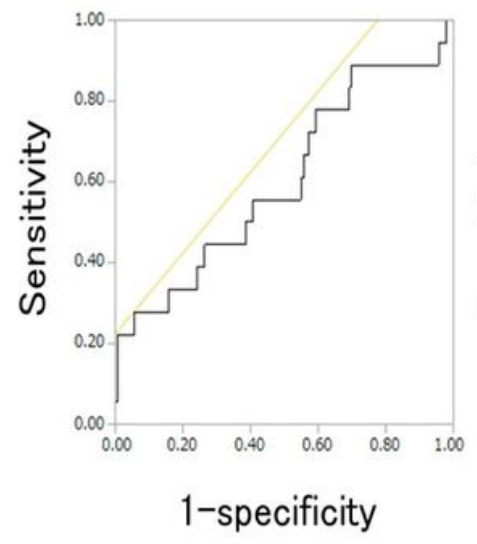

(b)

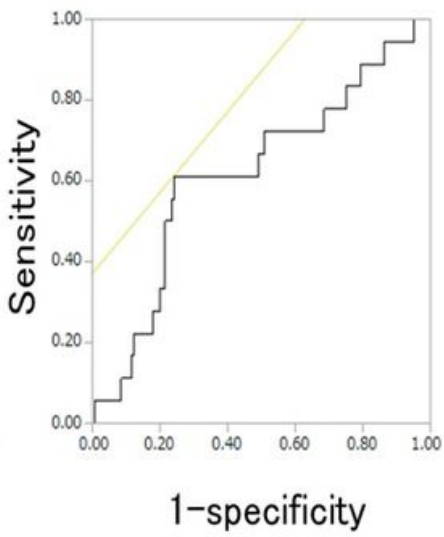

(c)

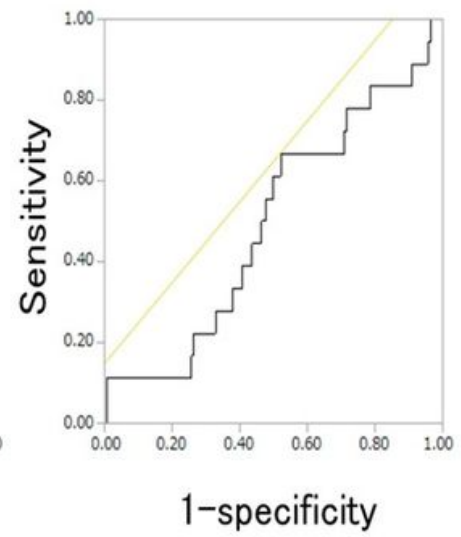

(d)

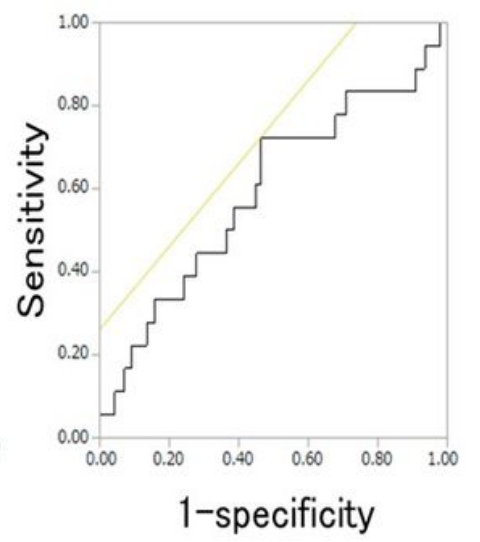

\section{Figure 1}

Receiver operating characteristics curve analysis of NLR (a), MLR (b), PLR (c), and PNI (d) for disease free survival.
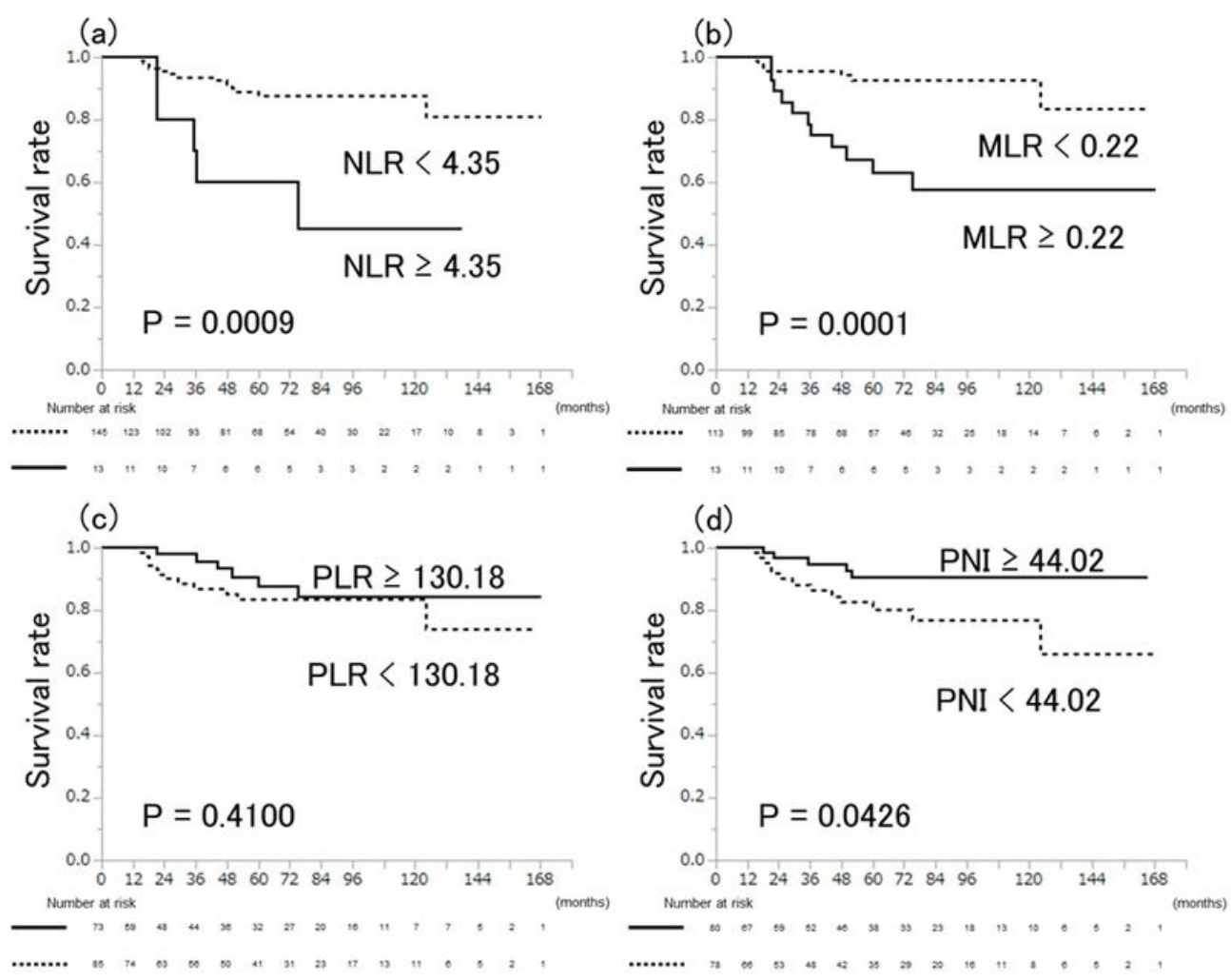
Figure 2

Kaplan-Meier curves for disease free survival according to the level of NLR (a), MLR (b), PLR (c), and PNI (d). 